\begin{tabular}{c|c|c|} 
SELECCIONES MATEMÁTICAS \\
Universidad Nacional de Trujillo \\
ISSN: $2411-1783$ (Online) \\
Vol. 06(01): $77-83(2019)$
\end{tabular}

\title{
Estabilidad casi segura de los sistemas lineales de salto Markoviano en tiempo discreto.
}

\section{Almost sure stability of discrete-time Markov jump linear systems. \\ Victor Camarena P. *}

Received, Dec. 31, 2017

Accepted, Apr. 13, 2019

DOI: http://dx.doi.org/10.17268/sel.mat.2019.01.10

\begin{abstract}
Resumen
El presente trabajo trata del estudio de la estabilidad casi segura de los sistemas lineales de salto Markoviano en tiempo discreto $\left(M J L S^{1}\right)$. Primero, se introduce la estabilidad de los sistemas lineales en tiempo discreto (MJLS con un solo modo) mediante el exponente de Lyapunov. Luego se aborda la extensión de dicha teoría al caso general: se hace uso de una variante del teorema ergódico de Birkhoff para revisar la igualdad (4.6) para el exponente de Lyapunov descrita en [6], a partir de esto se obtiene la caracterización de la estabilidad casi segura de los MJLS.
\end{abstract}

Palabras clave. Markov jump linear systems, exponente de Lyapunov, estabilidad casi segura.

\begin{abstract}
The present work deals with the study of the almost sure stability of discrete-time Markov jump linear systems $(M J L S)$. First, the stability of linear systems in discrete time (MJLS with a single mode) is introduced by the Lyapunov exponent. Then the extension of this theory to the general case is approached: a variant of Birkhoff's ergodic theorem is used to revise the equality (4.6) for the Lyapunov exponent described in [6], from this we obtain the characterization of the almost sure stability of the MJLS.
\end{abstract}

Keywords. Markov jump linear systems, Lyapunov exponent, almost sure stability.

1. Introducción. Consideremos el sistema de ecuaciones en diferencias

$$
x(k+1)=A x(k), \quad k \geq 0,
$$

donde $x(k) \in \mathbb{R}^{d}$ y $A$ es una matriz cuadrada de orden $d$. Recordemos que el sistema (1.1) es asintóticamente estable si para cualquier estado inicial $x(0) \in \mathbb{R}^{d}, \lim _{k \rightarrow \infty} x(k)=0$. La estabilidad de este sistema se puede caracterizar mediante el radio espectral de la matriz del sistema, $\rho(A)$ :

(1.1) es asintóticamente estable si y solo si $\rho(A)<1$.

Este resultado puede hallarse en el Resultado 1.3.9 de [9].

A partir de la fórmula del radio espectral, $\rho(A)=\operatorname{lím}_{k \rightarrow \infty}\left\|A^{k}\right\|^{1 / k}$, y de la continuidad y monotonía de la función ln se tiene que

$$
\lim _{k \rightarrow \infty} \frac{1}{k} \ln \left\|A^{k}\right\|=\ln \left(\lim _{k \rightarrow \infty}\left\|A^{k}\right\|^{1 / k}\right)=\ln \rho(A)<0 \Leftrightarrow \rho(A)<1 .
$$

Por lo tanto, se puede reescribir la caracterización anterior:

$$
\text { (1.1) es asintóticamente estable si y solo si } \lim _{k \rightarrow \infty} \frac{1}{k} \ln \left\|A^{k}\right\|<0,
$$

*ID ORCID: https://orcid.org/0000-0001-8024-4889, Facultad de Ciencias, Universidad Nacional de Ingeniería, Av. Túpac Amaru 210, Lima-Perú (vcamarenap@uni.pe)

This work is licensed under the Creative Commons Attribution-NoComercial-ShareAlike 4.0.

${ }^{1}$ Por sus siglas en inglés 
El límite $\lim _{k \rightarrow \infty} \frac{1}{k} \ln \left\|A^{k}\right\|$ es conocido como exponente de Lyapunov para el sistema (1.1).

Nuestro objetivo consiste en estudiar un problema más general conocido como sistema lineal de salto Markoviano en tiempo discreto el cual se describe mediante la dinámica

$$
x(k+1)=A_{\theta(k)} x(k), \quad k \geq 0,
$$

donde $x(k)$ es un vector en $\mathbb{R}^{d},\{\theta(k)\}_{k \geq 0}$ es una cadena de Markov con espacio de estados $\mathbb{S}=\{1,2, \ldots, N\}, \mathbb{N}$, definida sobre un espacio de probabilidad $(\Omega, \mathcal{F}, \mathbb{P})$. Para el sistema (1.2) y cada instante $k \geq 0$ : el vector $x(k)$ se denomina variable de estado del sistema, la matriz cuadrada $A_{\theta(k)}$ indica el modo de operación del sistema. Note que hay una cantidad finita o enumerable de modos de operación; y que a partir del instante $k=1$, en adelante, $x(k)$ es de naturaleza estocástica pues los modos de operación se están intercalando aleatoriamente, de acuerdo a la dinámica de transición de la cadena de Markov. Es por ello que este modelo matemático es útil para estudiar procesos que están sujetos a cambios estocásticos en su dinámica (modo de operación) pues cuando estas variaciones en el tiempo van afectando significativamente el comportamiento del sistema, bajo las hipótesis adecuadas, podemos cuantificar las probabilidades de los distintos escenarios posibles. Ejemplos de procesos de este tipo ocurren en sistemas económicos, sistemas de control de aeronaves, control de receptores centrales térmicos solares, sistemas manipuladores robóticos, grandes estructuras flexibles para estaciones espaciales, etc., donde los cambios abruptos pueden deberse a perturbaciones ambientales abruptas, fallos o reparaciones de componentes, cambios en las interconexiones de los subsistemas, cambios abruptos en el punto de funcionamiento de una instalación no lineal, etc. Un ejemplo que se puede revisar en la literatura es el problema de control de receptores centrales térmicos solares descrito en [10]. Nuestro propósito se centra en estudiar la estabilidad de los sistemas del tipo (1.2), como estos sistemas son de naturaleza estocástica hablar de estabilidad significa que casi todos los escenarios posibles, que son sistemas del tipo (1.1), son (asintóticamente) estables. Este concepto de estabilidad es conocido como estabilidad casi segura, y es la más débil de las estabilidades usuales definidas para los sistemas (1.2). En la siguiente sección veremos que bajo ciertas hipótesis para los parámetros del sistema (1.2) se obtiene una caracterización parcial de su estabilidad casi segura, como analogía de la caracterización ya conocida para el sistema (1.1).

2. La estabilidad casi segura de los sistemas lineales de salto Markoviano en tiempo discreto. Consideremos el sistema

$$
x(k+1)=A_{\theta(k)} x(k), \quad k \geq 0
$$

y la función ln : $[0,+\infty] \rightarrow[-\infty,+\infty]$ extendida de modo que se preserve como biyección creciente,

$$
\ln (x)=\left\{\begin{array}{cl}
-\infty & x=0 \\
\ln (x) & x \in] 0,+\infty[ \\
+\infty & x=+\infty
\end{array}\right.
$$

Asumimos las siguientes hipótesis:

$$
\begin{gathered}
G:=\sup _{i \in \mathbb{S}}\left\|A_{i}\right\|<+\infty \\
\sum_{i \in \mathbb{S}}\left|\ln \left\|A_{i}\right\|\right| \pi_{i}<+\infty,
\end{gathered}
$$

y la cadena de Markov $\{\theta(k)\}_{k>0}$ irreducible, ergódica, con matriz de transición $P$ y única distribución invariante $\pi$. Entonces, si $\theta(0) \sim \pi$ la cadena $\{\theta(k)\}_{k \geq 0}$ es estacionaria y de (2.3) se tiene que la cadena definida por

$$
f(\theta(k))=\ln \left\|A_{\theta(k)}\right\|
$$

verifica las hipótesis del teorema ergódico de Birkhoff para cadenas de Markov (ver Ejemplo 7.2.2 de [4]).

Definición 1. Decimos que el sistema (2.1) es casi seguramente estable (CSE) si para todas las condiciones iniciales $x(0)=x_{0} \in \mathbb{R}^{d}$ y $\theta(0)=\theta_{0} \sim \mu$, $\mu$ distribución en $\mathbb{S}$, se cumple

$$
\mathbb{P}_{\mu}\left(\lim _{k \rightarrow \infty}\|x(k)\|=0\right)=1 \text {. }
$$

Nuestra definición de estabilidad casi segura nos dice que hay convergencia al origen independientemente de las condiciones iniciales. Cuando la cadena de Markov $\{\theta(k)\}_{k \geq 0}$ es irreducible el Lema 2.3 de [6] muestra que para tener estabilidad casi segura basta verificar que

$$
\mathbb{P}_{\pi}\left(\lim _{k \rightarrow \infty}\|x(k)\|=0\right)=1,
$$


para cualquier vector de estado inicial $x_{0} \in \mathbb{R}^{d}$. Esto motiva la siguiente definición.

Definición 2. El exponente de Lyapunov para el sistema (2.1) se define mediante la fórmula

$$
\alpha_{\pi}:=\lim _{k \rightarrow \infty} \frac{1}{k} \mathbb{E}_{\pi}\left\{\ln \left\|A_{\theta(k-1)} \ldots A_{\theta(0)}\right\|\right\} .
$$

Este es el más grande exponente de Lyapunov que se puede definir para el sistema (2.1) y por el comentario previo cuando la cadena es irreducible permitirá caracterizar la estabilidad casi segura. La prueba del siguiente lema que garantiza la buena definición del exponente de Lyapunov consiste en usar la propiedad subaditiva de la sucesión

$$
a_{k}=\mathbb{E}_{\pi}\left\{\ln \left\|A_{\theta(k-1)} \ldots A_{\theta(0)}\right\|\right\}
$$

y seguir sin cambio alguno los mismos pasos del Lema 4.5 de [6].

Lema 1. El exponente de Lyapunov está bien definido, además se cumple

$$
\alpha_{\pi}=\inf _{k \geq 1} \frac{1}{k} \mathbb{E}_{\pi}\left\{\ln \left\|A_{\theta(k-1)} \ldots A_{\theta(0)}\right\|\right\} .
$$

El siguiente lema nos da una forma práctica del exponente de Lyapunov para facilitar su aplicación (Esta es la igualdad 4.6 de [6]).

Lema 2. El exponente de Lyapunov $\alpha_{\pi}$ se puede expresar

$$
\alpha_{\pi}=\underset{k}{\limsup } \frac{1}{k} \ln \left\|A_{\theta(k-1)} \ldots A_{\theta(0)}\right\| \quad \mathbb{P}_{\pi}-\text { c.s. }
$$

Demostración: La prueba sigue los mismos lineamientos dados en el Lema 4.5 de [6]. Para cada $l \geq 1$ definimos la cadena

$$
\theta^{l}(k)=(\theta((k+1) l-1), \ldots, \theta(k l)), k \geq 0
$$

la cual es Markoviana (con matriz de transición $\tilde{P}^{l}$ asociada a $P$ ), de tiempo homogéneo, irreducible, con distribución inicial igual a su única distribución invariante $\pi^{l}$ (asociada a $P$ y $\pi$ ), y por lo tanto es estacionaria y todos sus estados son recurrentes positivos.

Además, definimos el evento

$$
A:=\left\{\omega \in \Omega: \exists k \geq 1 / A_{\theta(k-1, \omega)} \ldots A_{\theta(0, \omega)}=0\right\} .
$$

i) Si $\mathbb{P}_{\pi}(A)>0$. De la prueba de la buena definición del exponente de Lyapunov (lema 1) para este caso se tiene $\alpha_{\pi}=-\infty$. Por otro lado, para algún $l_{0} \geq 1$ se cumple

$$
\mathbb{P}_{\pi}\left(\omega \in \Omega: A_{\theta\left(l_{0}-1, \omega\right)} \ldots A_{\theta(0, \omega)}=0\right)>0,
$$

así existe un estado $j^{0}=\left(j_{l_{0}-1}, \ldots, j_{0}\right)$ de la cadena $\theta^{l_{0}}$ tal que $A_{j_{l_{0}-1}} \ldots A_{j_{0}}=0$. Como $j^{0}$ es recurrente positivo se obtiene que con $\mathbb{P}_{\pi}$-casi seguramente,

$$
A_{\theta(k-1)} \ldots A_{\theta(0)}=0
$$

para $k$ suficientemente grande. Así, con $\mathbb{P}_{\pi}$-casi seguramente

$$
\limsup _{k} \frac{1}{k} \ln \left\|A_{\theta(k-1)} \ldots A_{\theta(0)}\right\|=-\infty .
$$

ii) Si $\mathbb{P}_{\pi}(A)=0$. Entonces con $\mathbb{P}_{\pi}$-casi seguramente se tiene que $A_{\theta(k-1)} \ldots A_{\theta(0)} \neq 0$ para cualquier $k \geq 1$, y por lo tanto

$$
\ln \left\|A_{\theta(k-1)} \ldots A_{\theta(0)}\right\|>\infty .
$$


Sea $l \geq 1, k=p l+q$ con $0 \leq q<l$, luego

$$
\begin{aligned}
& \frac{1}{k} \ln \left\|A_{\theta(k-1)} \ldots A_{\theta(0)}\right\|=\frac{1}{k} \ln \left\|A_{\theta(k-1)} \ldots A_{\theta(p l)}\right\|+\frac{1}{k} \ln \left\|A_{\theta(p l-1)} \ldots A_{\theta(0)}\right\| \\
& \leq \frac{1}{k} \ln G^{k-p l}+\frac{1}{k} \sum_{n=0}^{p-1} \ln \left\|A_{\theta((n+1) l-1)} \ldots A_{\theta(n l)}\right\| \\
& \leq \frac{1}{k} \ln G^{q}+\frac{p}{k}\left(\frac{1}{p} \sum_{l=0}^{p-1} \ln \left\|A_{\theta((n+1) l-1)} \ldots A_{\theta(n l)}\right\|\right) \\
& \leq \frac{q}{k} \ln G+\frac{1}{l}\left(\frac{1}{p} \sum_{l=0}^{p-1} \ln \left\|A_{\theta((n+1) l-1)} \ldots A_{\theta(n l)}\right\|\right)
\end{aligned}
$$

La cadena de Markov $\theta^{l}(n)=(\theta((n+1) l-1), \ldots, \theta(n l)), n \geq 0$, es estacionaria y verifica las hipótesis del teorema ergódico de Birkhoff, entonces

$$
\frac{1}{p} \sum_{n=0}^{p-1} \ln \left\|A_{\theta((n+1) l-1)} \ldots A_{\theta(n l)}\right\| \rightarrow \mathbb{E}_{\pi^{l}}\left\{\ln \left\|A_{\theta(m-1)} \ldots A_{\theta(0)}\right\|\right\}
$$

en $L^{1}$ y $\mathbb{P}_{\pi^{l}}$-casi seguramente; más aún, la esperanza y convergencia casi seguramente son las mismas para la probabilidad $\mathbb{P}_{\pi}$. Entonces, tomando límite superior a la expresión (5), cuando $p \rightarrow \infty$, se sigue que $\mathbb{P}_{\pi}$-casi seguramente

$$
\underset{k}{\limsup } \frac{1}{k} \ln \left\|A_{\theta(k-1)} \ldots A_{\theta(0)}\right\| \leq 0+\frac{1}{l} \mathbb{E}_{\pi}\left\{\ln \left\|A_{\theta(l-1)} \ldots A_{\theta(0)}\right\|\right\}
$$

Por consiguiente, del lema $1, \mathbb{P}_{\pi}$-casi seguramente

$$
\limsup _{k} \frac{1}{k} \ln \left\|A_{\theta(k-1)} \ldots A_{\theta(0)}\right\| \leq \inf _{l \geq 1} \frac{1}{l} \mathbb{E}_{\pi}\left\{\ln \left\|A_{\theta(l-1)} \ldots A_{\theta(0)}\right\|\right\}=\alpha_{\pi} .
$$

Definimos auxiliarmente la sucesión de variables aleatorias no negativas, para $k \geq 1$ sea

$$
\gamma_{k}=\frac{1}{k} \sum_{n=0}^{k-1} \ln \left\|A_{\theta(n)}\right\|-\frac{1}{k} \ln \left\|A_{\theta(k-1)} \ldots A_{\theta(0)}\right\| .
$$

Por el teorema ergódico de Birkhoff para la cadena de Markov estacionaria $\theta(n), n \geq 0$, se consigue

$$
\frac{1}{k} \sum_{n=0}^{k-1} \ln \left\|A_{\theta(n)}\right\| \rightarrow \mathbb{E}_{\pi}\left\{\ln \left\|A_{\theta(0)}\right\|\right\}
$$

en $L^{1}$ y $\mathbb{P}_{\pi}$-casi seguramente. Entonces, tomando límite inferior, cuando $k \rightarrow \infty$, a la sucesión $\gamma_{k}$ se tiene que $\mathbb{P}_{\pi}$-casi seguramente

$$
\liminf _{k} \gamma_{k}=\mathbb{E}_{\pi}\left\{\ln \left\|A_{\theta(0)}\right\|\right\}-\limsup _{k} \frac{1}{k} \ln \left\|A_{\theta(k-1)} \ldots A_{\theta(0)}\right\|
$$

y, como $\mathbb{E}_{\pi} \gamma_{k}=\mathbb{E}_{\pi}\left\{\ln \left\|A_{\theta(0)}\right\|\right\}-\frac{1}{k} \mathbb{E}_{\pi}\left\{\ln \left\|A_{\theta(k-1)} \ldots A_{\theta(0)}\right\|\right\}$, también se tiene que

$$
\liminf _{k} \mathbb{E}_{\pi} \gamma_{k}=\mathbb{E}_{\pi}\left\{\ln \left\|A_{\theta(0)}\right\|\right\}-\alpha_{\pi} .
$$

De este último par de límites, (2.7) y (2.8), y aplicando el lema de Fatou a la sucesión $\gamma_{k}$ se sigue

$$
\begin{aligned}
\mathbb{E}_{\pi}\left\{\ln \left\|A_{\theta(0)}\right\|\right\}-\alpha_{\pi}=\liminf _{k} \mathbb{E}_{\pi} \gamma_{k} & \geq \mathbb{E}_{\pi}\left\{\operatorname{líminf}_{k} \gamma_{k}\right\} \\
& =\mathbb{E}_{\pi}\left\{\ln \left\|A_{\theta(0)}\right\|\right\}-\mathbb{E}_{\pi} \limsup _{k} \frac{1}{k} \ln \left\|A_{\theta(k-1)} \ldots A_{\theta(0)}\right\|,
\end{aligned}
$$

es decir,

$$
\alpha_{\pi} \leq \mathbb{E}_{\pi}\left\{\limsup _{k} \frac{1}{k} \ln \left\|A_{\theta(k-1)} \ldots A_{\theta(0)}\right\|\right\}
$$


Por lo tanto, de (2.6) y (2.9), se cumple que $\mathbb{P}_{\pi}$-casi seguramente

$$
\alpha_{\pi}=\underset{k}{\limsup } \frac{1}{k} \ln \left\|A_{\theta(k-1)} \ldots A_{\theta(0)}\right\| .
$$

A continuación se establece una caracterización parcial de la estabilidad casi segura del sistema (2.1).

Teorema 1. El exponente de Lyapunov caracteriza la estabilidad del sistema (2.1):

i) Si $\alpha_{\pi}<0$ entonces (2.1) es casi seguramente estable.

ii) Si $\alpha_{\pi}>0$ entonces (2.1) no es casi seguramente estable.

Demostración: i) Tenemos que $\alpha_{\pi}<0$, es decir, del lema 2,

$$
\underset{k}{\limsup } \frac{1}{k} \ln \left\|A_{\theta(k-1)} \ldots A_{\theta(0)}\right\|=\alpha_{\pi}<0 \quad \mathbb{P}_{\pi}-\text { c.s. }
$$

Por definición de límite superior tenemos que para $\alpha_{\pi}<c<0$, fijo, existe $k_{0} \geq 1$ suficientemente grande tal que

$$
\sup _{k \geq k_{0}}\left\{\frac{1}{k} \ln \left\|A_{\theta(k-1)} \ldots A_{\theta(0)}\right\|\right\}<c \quad \mathbb{P}_{\pi}-c . s .
$$

de donde $\mathbb{P}_{\pi}$-casi seguramente

$$
\left\|A_{\theta(k-1)} \ldots A_{\theta(0)}\right\| \leq e^{k c} \forall k \geq k_{0}
$$

entonces, para cualquier $x(0) \in \mathbb{R}^{d}$ se tiene que $\mathbb{P}_{\pi}$-casi seguramente

$$
\limsup _{k \geq k_{0}}\|x(k)\|=\limsup _{k \geq k_{0}}\left\|A_{\theta(k-1)} \ldots A_{\theta(0)} x(0)\right\| \leq \limsup _{k \geq k_{0}} e^{k c}\|x(0)\|=0 .
$$

Por lo tanto, se verfica (2.4) y el sistema (2.1) es casi seguramente estable.

ii) Probaremos el contrarecíproco. Por hipótesis, en particular para el sistema (2.1) se verifica (2.4), lo cual implica la siguiente afirmación

$$
\mathbb{P}_{\pi}\left(\lim _{k \rightarrow \infty}\left\|A_{\theta(k-1)} \ldots A_{\theta(0)}\right\|=0\right)=1
$$

Para esta última afirmación basta poner $x(0)=e_{i} \in \mathbb{R}^{d}$ con $i=1, \ldots, d$, y darse cuenta que esto implica que, $\mathbb{P}_{\pi}$-casi seguramente, cada vector columna de $A_{\theta(k-1)} \ldots A_{\theta(0)}$ converge a cero, cuando $k \rightarrow \infty$.

Así, $\mathbb{P}_{\pi}$-casi seguramente,

$$
\left\|A_{\theta(k-1)} \ldots A_{\theta(0)}\right\|<1, \forall k \geq k_{0}
$$

para $k_{0}$ suficientemente grande, de donde calculando el límite superior como en el lema 2, se obtiene

$$
\limsup _{k} \frac{1}{k} \ln \left\|A_{\theta(k-1)} \ldots A_{\theta(0)}\right\| \leq \ln 1=0 .
$$

Por lo tanto $\alpha_{\pi} \leq 0$. $\square$

Ejemplo 1. Sea $\{\theta(k)\}_{k>0}$ una cadena de Markov que toma solo dos estados, irreducible, recurrente y con distribución invariante $\pi=(0,5,0,5)$, matriz de transición

$$
P=\left(\begin{array}{cc}
0,5 & 0,5 \\
0,5 & 0,5
\end{array}\right)
$$

Sean los dos posibles modos de operación

$$
A_{1}=\left(\begin{array}{ll}
2 & 0 \\
0 & 0
\end{array}\right), \quad A_{2}=\left(\begin{array}{ll}
0 & 0 \\
0 & 2
\end{array}\right) .
$$

Notemos que $A_{1} A_{2}=A_{2} A_{1}=0$. Como en la prueba del lema 2 definimos la siguiente cadena de Markov $\tilde{\theta}(k)=(\theta(2 k+1), \theta(2 k)), k \geq 0$, con espacio de estados

$$
\{\tilde{1}=(1,1), \tilde{2}=(1,2), \tilde{3}=(2,1), \tilde{4}=(2,2)\},
$$


y matriz de transición

$$
\tilde{P}=\left(\begin{array}{llll}
0,25 & 0,25 & 0,25 & 0,25 \\
0,25 & 0,25 & 0,25 & 0,25 \\
0,25 & 0,25 & 0,25 & 0,25 \\
0,25 & 0,25 & 0,25 & 0,25
\end{array}\right)
$$

Por lo tanto, la cadena es irreducible, recurrente positiva, y tiene distribución invariante

$$
\tilde{\pi}=(0,25,0,25,0,25,0,25)
$$

Así, como el estado $\tilde{2}=(1,2)$ es recurrente positivo, $\mathbb{P}_{\tilde{\pi}}$-casi seguramente este estado aparece en una secuencia $(\theta(k-1), \ldots, \theta(0))$ para $k$ suficientemente grande. Luego, $\mathbb{P}_{\tilde{\pi} \text {-casi seguramente }}$ $\ln \left\|A_{\theta(k-1)} \ldots A_{\theta(0)}\right\|=-\infty$ para $k$ suficientemente grande. Así se consigue $\alpha_{\pi}=-\infty<0$ y el sistema es $\mathbb{P}_{\pi}$-casi seguramente. Observe que cada modo de operación es inestable pues $\rho\left(A_{1}\right)=\rho\left(A_{2}\right)=2 \geq 1$, es decir, la estabilidad del sistema de salto Markoviano no implica la estabilidad de los sistemas invariantes asociados a un solo modo de operación. Para una mayor discusión de esta anomalía ver el Ejemplo 2.9.4 de [5] donde se hace un estudio de sistemas con modos de operación más genéricos aunque sin dependencia para las transiciones entre estos.

Ahora, se tiene un tipo de estabilidad casi segura más fuerte que nos dice que la convergencia al origen es de orden exponencial. Aquí se muestra su relación con la estabilidad casi segura.

Definición 3. Decimos que el sistema (2.1) es casi segura exponencialmente estable (CSEE) si para todas las condiciones iniciales $x(0)=x_{0} \in \mathbb{R}^{d}$ y $\theta(0)=\theta_{0} \sim \mu$, $\mu$ distribución en $\mathbb{S}$, existe $\gamma>0$ tal que

$$
\mathbb{P}_{\mu}\left(\underset{k}{\operatorname{límsup}} \frac{1}{k} \ln \|x(k)\| \leq-\gamma\right)=1 \text {. }
$$

Está definición aparece en el artículo [2]. No difícil mostrar que si el sistema (2.1) es CSEE entonces también es CSE, sin embargo, el recíproco no es cierto en general como lo muestra el siguiente ejemplo.

Ejemplo 2. Cuando la cadena de Markov $\{\theta(k): k \geq 0\}$ toma valores en un espacio de estados numerable, los conceptos de CSEE y CSE no son equivalentes. De hecho, considere el sistema (2.1) con d = 1,

$$
P_{i, i+1}=1 \quad y \quad A_{i}=\frac{i}{i+1}, \quad i=1,2,3, \ldots
$$

En este caso

$$
\|x(k)\|=\frac{\theta_{0}}{\theta_{0}+k}\left|x_{0}\right| \rightarrow 0 \quad c . s .,
$$

cuando $k \rightarrow \infty$, para cualesquiera condiciones iniciales $\theta_{0} \sim \mu$, $\mu$ distribución en $\mathbb{N}, x_{0} \in \mathbb{R}^{d}$, es decir, el sistema es CSE. Sin embargo, cuando $x_{0}=1$ y $\theta_{0}=1$ se tiene que

$$
\frac{1}{k} \ln \|x(k)\|=\frac{1}{k} \ln \frac{1}{1+k}=-\frac{k+1}{k} \frac{\ln (k+1)}{k+1} \rightarrow 0 \quad \text { c.s. }
$$

es decir, el sistema no es CSEE.

Es importante hacer notar que dado del comportamiento de un sistema (1.1), uno esperaría que se pueda obtener una caracterización de la estabilidad casi segura de un sistema (2.1) por medio del exponente de Lyapunov, lo cual nos lo dice parcialmente el Teorema 1. Siendo más acertados en nuestra intuición uno debe esperar que el exponente de Lyapunov caracterize la estabilidad exponencial casi segura, lo cual está guardado en la discusión hecha en [7]. La prueba de esta caracterización explota la idea de construir una cadena de Markov como se hizo en el Lema 2, esto puede hallarse en [2] donde además diseñan un algoritmo para testear la estabilidad exponencial casi segura.

3. Conclusiones. De nuestro trabajo se concluye que el exponente de Lyapunov permite caracterizar la estabilidad casi segura (CSE) para los sistemas lineales de salto Markoviano solo determinando el signo que este toma. Sin embargo, se tiene que la caracterización es completa cuando se considera la estabilidad exponencial casi segura (CSEE).

4. Agradecimientos. El autor agradece de forma especial a la Universidad Nacional de Trujillo por ofrecer la oportunidad de presentar el trabajo en el XI Fast Worshop on Applied and Computational Mathematics realizado en la ciudad de Trujillo del 04 al 05 de enero del 2018. 


\section{Referencias}

[1] Birkhoff, G. D., Proof of the Ergodic Theorem. Proceedings of the National Academy of Sciences of the United States of America. 1931; 17(12): 656-660. doi 10.1073/pnas.17.2.656

[2] Bolzern, P., Colaneri, P. and Nicolao, G. D., On almost sure stability of discrete-time markov jump linear systems. In 2004 43rd IEEE Conference on Decision and Control (CDC) (IEEE Cat. N 04CH37601). 2004; 3: 3204-3208. doi 10.1109/CDC.2004.1428966

[3] Costa, O.L.V., Fragoso, M.D. and Marques, R.P., Discrete-time Markov jump linear systems. Springer-Verlag London, 2005.

[4] Durrett, R., Probability: theory and examples. Cambridge University Press, 2010.

[5] Fang, Y., Stability Analysis of Linear Control Systems with Uncertain Parameters [Ph.D. thesis], Case Western Reserve University, Ohio, 1994. Recuperado de http://www.fang.ece.ufl.edu/mypaper/cwru.pdf

[6] Fang, Y., Loparo and Feng, X., Stability of discrete time jump linear systems. Journal of Mathematical Systems, Estimation and Control. 1995; 5(3): 275-321. Recuperado de http://www.fang.ece.ufl.edu/mypaper/jmsec95fang.pdf

[7] Ishii, H., Discussion on: "Almost Sure Stability of Stochastic Linear Systems with Ergodic Parameters". Eur. J. Control. 2008; 14: 125-127. doi 10.1016/S0947-3580(08)70754-4

[8] Norris, J. R., Markov chains. Cambridge University Press, 1998

[9] Ortega, J. M., Numerical analysis: a second course. SIAM, 1990.

[10] Sworder, D. D. and Rogers, R. O., An LQG solution to a control problem with solar thermal receiver. IEEE Transactions on Automatic Control. 1983; 28: 971-978. doi 10.1109/TAC.1983.1103151 